\title{
Splicing Forgery Exposure in Digital Image by Detecting Noise Discrepancies
}

\author{
Bo Liu and Chi-Man Pun
}

\begin{abstract}
We propose a novel technique in this paper to expose splicing forgery in digital images. The forged area spliced from other picture contains some features which may be inconsistent with the rest part of image. Noise pattern and level is a possible factor to reveal such inconsistency. To detect such noise discrepancies, the test picture is initially segmented into small pieces by SLIC superpixels algorithm. The noise pattern and level of each segment is then estimated by using various filters. The noise features constructed in this step are utilized in energy-based graph cut to expose forged area in the final step. Experimental results show that our method provides good illustration of regions with noise inconsistency in various scenarios.
\end{abstract}

Index Terms-Forgery detection, graphic cuts, noise estimation, SLIC, splicing forgery.

\section{INTRODUCTION}

To determine whether a particular picture has been intentionally manipulated or not is a principle problem in multimedia forensics. Some researchers make a hypothesis that the spliced region which does not originally belong to the picture will be altered in terms of dimensions, shape, color or illumination in order to fit the host picture. Mahdian et al. [1] noticed that interpolated signals and their derivatives contain specific detectable periodic properties. And geometric transformations, such as scaling, rotation or skewing of spliced objects involve resampling and interpolation steps. Thus these traces can be detected. Similar approach was also proposed in [2]. The weakness of these methods is that they only show good performance to images in TIFF format and are not robust to JPEG compression. The other main problem is these proposed methods are incapable of handling the situation that spliced regions are not undergone geometrical changes.

To solve the problem, for instance, motion blur discrepancies [3] are used as a forensic indicator. Estimation of the quantization matrix in JPEG compression algorithm provides another way to detect modification traces. Proposed by Hamdy et al. [4] some DCT coefficients which are used in JPEG compression process of an image are estimated and the regions possess different coefficients are suspected as splicing area. However, the algorithm cannot deal with double compression. Practically this method only detect BMP-format picture which is spliced of two JPG images with different compression table.

Manuscript received May 14, 2014; revised August 30, 2014.

The authors are with the Department of Computer and Information Science, University of Macau, Macau, China (e-mail: liubo.macau@gmail.com,cmpun@umac.mo).
Noise patterns and levels are important features can also be employed in inconsistency analysis. If a photo is taken by a digital camera, noise will inevitably effect the image quality. The noise comes from imaging sensor and internal circuits within a camera. And the number of noise changes in accordance with camera settings especially ISO sensitivity and exposure time. And the noise reduction algorithm differs among different manufactures, thus noise pattern and level varies from different pictures. Moreover the propagation may add new noise and people can also add or reduce noise for certain purpose as well. So the noise can be used as the feature of an image. When two pictures are spliced together the noise level or patterns are inconsistent between regions. By estimating the pattern or level of noise in different regions the forgery can be exposed via noise discrepancies. Prior work has been made by Guo et al. [5] but they only provided simulation results rather than actual fake image forensics and did not demonstrate the valid authentication results. While we will discuss and show it in details in the paper.

\section{NOISE FEATURE EXTRACTION}

The flow chart of the method is shown in Fig. 1. The first step is to extract noise feature. As stated above the noise of image taken from camera is inevitable while the amount and noise changes according to camera settings and the different manufacture. As an example Fig. 2 shows the visual noise of images captures from a Nikon D7000 camera. We can see more noise appears in the image as the ISO speed rises. In Fig. 3 we can see different camera model from different manufacture also shows unequal noise amount and forms although the pictures were taken in the same scenery with equal ISO speed. These facts make it possible to exposure splicing forgeries by detecting noise discrepancies. In this section, the first step, noise feature extraction will be investigated.

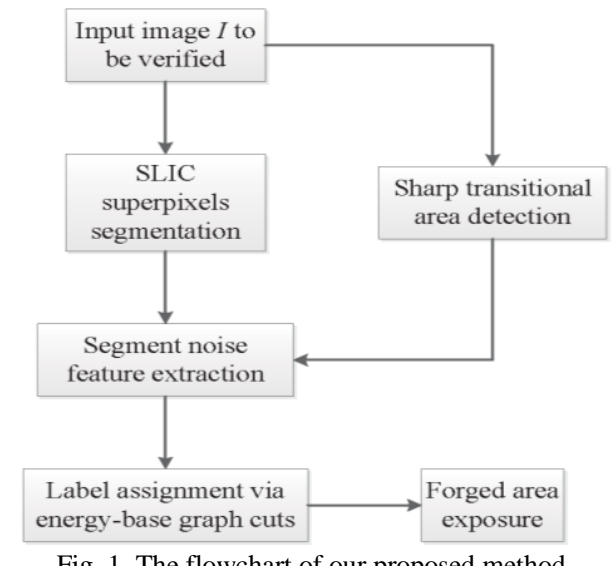

Fig. 1. The flowchart of our proposed method. 
In most cases, the alien region has a specific shape, such as a tree, a bird or a person. The forged object may possess different noise level comparing to that of its surroundings. To estimate every region's noise level, the image should be firstly divided into small segments. Most previous methods divides the picture into small overlapping blocks with equal size. But in our application, this means will lead to bad performance in next step which need accurate noise estimation of each region to compare noise discrepancy. This is because the forged area is not rectangle in most cases, and the small block will contain original as well as alien pixels. Therefore we segment picture into sets of pixels, not regular shaped, also known as superpixels. Employing this approach makes segments more meaningful and easier to be process in the following steps because the segmentation algorithms locate the objects and boundaries other than same-size blocks. The output of superpixels segmentation is some segments which cover the whole image. Pixels in a region are similar with respect to some properties, such as color or intensity. Adjacent regions are substantially different with respect to these characteristic(s) [6].



(a)

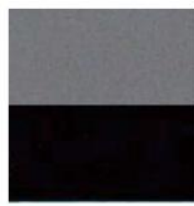

(b)

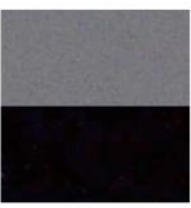

(c)



(d)
Fig. 2. Visual noise comparison for pictures captured by same camera Nikon D7000 under the same scenery with different ISO settings. (a) ISO $=100$; (b) $\mathrm{ISO}=800$; (c) $\mathrm{ISO}=1600$; (d) $\mathrm{ISO}=3200$. Crops are $100 \%$ with ambient temperature approximately $22^{\circ} \mathrm{C}$. The experimental result is available on www.dpreview.com.

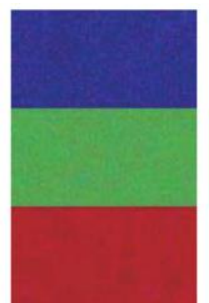

(a)



(b)



(c)

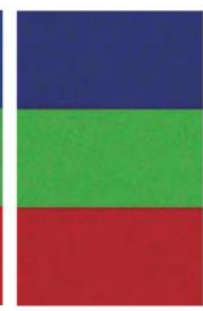

(d)
Fig. 3. Visual noise comparison for pictures taken by different cameras under the same scenery with ISO=1600. (a) Canon 550D; (b) Nikon D7000; (c) Sony A77; (d) Pentax K5. The experimental result is available on www.dpreview.com.

In our application, SLIC (Simple Linear Iterative Clustering) superpixels algorithm [7] was used to segment picture. This algorithm is easy but better than other segmentation methods. Given an $M \times N$ image $I_{s}^{c}$, where $c \in\{$ red, green,blue $\}$ denotes different color channel. The meaning of subscript $s$ will be explained later and

$$
I=\left(\begin{array}{ccc}
I_{s}^{c}(1,1) & \ldots & I_{s}^{c}(1, N) \\
\vdots & \ddots & \vdots \\
I_{s}^{c}(M, 1) & \cdots & I_{s}^{c}(M, N)
\end{array}\right)
$$

In essence, SLIC is a clustering algorithm. Similar to other clustering methods, two steps are evolved with SLIC segmentation. In the initialization step cluster centers are assigned by sampling pixels at regular grid. In the assignment step, each pixel is associated with the nearest cluster center and an update step adjusts the cluster centers to be the mean vector of all the pixels belonging to the cluster. The segmented picture is shown in Fig. 4. We assigned subscript $s$ which denoted segment number to every pixel.

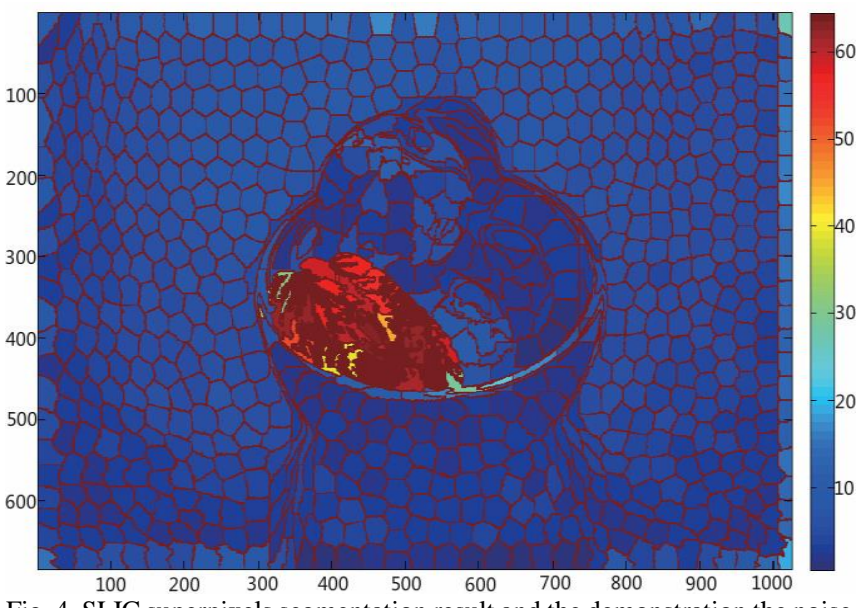

Fig. 4. SLIC superpixels segmentation result and the demonstration the noise level of each segment. The original picture is Fig. 7(c).

Before construction of noise feature for every segment, we excluded sharp transitional area since noise estimation was adversely affected by heterogeneous image content [8]. We estimated sharp area using its gray-scale image $G$. The sharpness edge of image was then obtained by $E=G^{*} S$, where $S$ represents Sobel operator and “*” denotes convolution. We then define whether a pixel is in the sharp area using

$$
B(m, n)= \begin{cases}1 & (m, n) \in E \\ 0 & (m, n) \notin E\end{cases}
$$

where $B(m, n)=1$ means the pixel $(m, n)$ locates in sharp transitional area. To guarantee these areas will not affect noise estimation in the next step, we expand boundaries via dilation by $L=B \oplus V$, where $V$ is a structure element of $3 \times 3$ ones. $L$ is the expanded sharp area. See Fig. 5.

To extract noise feature of each segment produced by previous SLIC algorithm, we firstly employed denoising algorithm across the whole picture. The estimated noise $f$ at location $(m, n)$ of image $I^{c}$ was calculated by $f^{c d}(m, n)=I^{c}(m, n)-D^{c d}(m, n)$, where $D^{c d}=I^{c} * P^{d}$, and filter $P^{d}, d=1,2, \ldots, 5$ represents five different filters used to trace different aspects of the noise [9]. They are median filter, Gaussian filter, averaging filter and adaptive Wiener denoising with two neighborhood sizes $3 \times 3$ and $5 \times 5$ respectively. For instance, high frequency noise can be detected by using Gaussian filter and median filter addresses "salt and pepper" noise.

For each combination of color channel $c$ and denoising filter $P^{d}$ we calculated the mean $\mu_{s}^{c d}$ and standard deviation $\sigma_{s}^{c d}$ values of each segment $s$ as the noise feature $F_{s}^{c d}=\left(\mu_{s}^{c d}, \sigma_{s}^{c d}\right)$, where 


$$
\mu_{s}^{c d}=\frac{1}{R} \sum_{L(m, n)=0} f_{s}^{c d}(m, n)
$$

And

$$
\sigma_{s}^{c d}=\frac{1}{R}\left(\sum_{L(m, n)=0}\left(f_{s}^{c d}(m, n)-\mu_{s}^{c d}\right)^{2}\right)^{\frac{1}{2}} .
$$

As a result we computed $3 \times 5 \times 2=30$ dimensional feature vector $F_{s}$ of a segment.
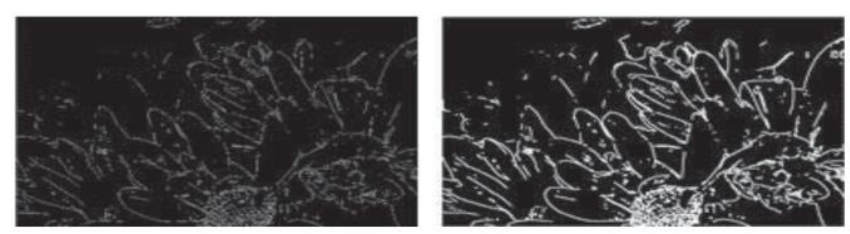

Fig. 5. Sharp transitional area detection and dilation. Left: Edge detection result; right: dilation result.

\section{DETECTING NOISE DisCREPANCIES VIA GRAPH CUTS}

Till now the noise feature of each segment have been calculated and then our goal is to segment the image into two regions which exhibit noise discrepancies. To achieve the target the energy-base graph cuts can be used.

\section{A. A Brief Introduction to Energy-Based Graph Cuts}

Energy minimization via graph cuts is proposed by Boykov et al. [10] to solve labeling problems with low computation cost. In a common label assignment problem, the labels should change smoothly cross the whole image while preserving sharp discontinuities at boundaries of objects. These two constrains can be formulated as

$$
E(f)=E_{\text {smooth }}(f)+E_{\text {data }}(f) .
$$

where $f$ is a labeling that assigns each pixel $p \in P$ a label $f_{p} \in L$, and $E_{\text {smooth }}$ measures the extent to which $f$ is not piecewise smooth while $E_{\text {data }}$ measures the disagreement between $f$ and observed data. The goal is to minimize the function. Specifically the energy function can be rewrote as the form

$$
E(f)=\sum_{\{p, q\} \in N} V_{p, q}\left(f_{p}, f_{q}\right)+\sum_{p \in P} D_{p}\left(f_{p}\right)
$$

where $N$ is neighboring pixels and $V$ is the penalty of pairs in the first term and $D_{p}$ is nonnegative and measures how well label fits pixel. Local minimum value can be obtained with the help of graph cuts. The simplified problem is illustrated in Fig. 6. Since many algorithms have been proposed to solve min-cut problem, if proper weight value is assigned to each edge, the problem of minimizing energy function changes to min-cut problem. The weight is seen in Table I. The calculation result is a cut $C$ which separates two labels. Fig. 6 shows two possible cuts and the label is assigned to the pixel when cut $C$ contains the edge connecting that label to the pixel. For example, in left case of Fig. 6, label $\alpha$ is assigned to pixel $p$ while $\beta$ is assigned to $q$ because cut

$C$ contains edge $t_{p}^{\alpha}$ and $t_{q}^{\beta}$.
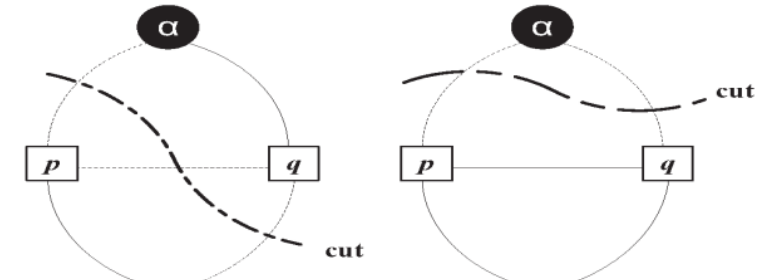

B



Fig. 6. Two possible graph cuts result. $\alpha, \beta$ are two labels and $p, q$ are pixels

TABLE I: EDGE WEIGHTS FOR GRAPH CUTS

\begin{tabular}{ccc}
\hline \hline \multicolumn{3}{c}{ TABLE I: EDGE WEIGHTS FOR GRAPH CUTS } \\
\hline Edge & Weight & For \\
\hline$t_{p}^{\alpha}$ & $D_{p}(\alpha)+\sum_{\substack{q \in N_{p} \\
q \notin P_{\alpha \beta}}} V\left(\alpha, f_{q}\right)$ & $p \in P_{\alpha \beta}$ \\
\hline$t_{p}^{\beta}$ & $D_{p}(\beta)+\sum_{\substack{q \in N_{p} \\
q \notin P_{\alpha \beta}}} V\left(\beta, f_{q}\right)$ & $p \in P_{\alpha \beta}$ \\
\hline$e_{\{p, q\}}$ & $V(\alpha, \beta)$ & $\{p, q\} \in N$ \\
\hline \hline
\end{tabular}

\section{B. Label Assignment for Segments}

Our forgery detection task can also be regarded as a labeling problem. In our application, there are two labels need to be assigned to each segment: forged area and the original area. The reasons why we avoid employing widely used outlier detection algorithms [11] and Otsu's automatic thresholding method [12] is the property of noise. From Fig. 2 we observe that even the picture is taken by one camera, the amount of noise differs in different illumination. The color of object may also affect the noise level. Accordingly the ideal algorithm should tolerate these local deviations and inconsistencies. This requirement is identical to label assignment problems described previously while normal outlier detection algorithms are not capable.

"Smooth" constrain is realized by proper assignment of $V(\alpha, \beta)$ and "sharp" discontinues requirement is supported by $D_{p}(*)$. We firstly discuss about the weight of edge $t_{p}^{\alpha}$ and $t_{p}^{\beta}$. We computed average value of feature vector of all segments in 30 dimensions and named it the mean vector $\bar{F}$. Then we found the vector whose Euclidean distance was largest from $\bar{F}$ by searching all segments and called it $F_{\text {max }}$. For a feature vector $F_{s}$ the weight $w$ was obtained by $w_{\alpha}=\left\|F_{s}-\bar{F}\right\|$ and $w_{\beta}=\left\|F_{s}-F_{\max }\right\|$, where $\alpha$ was “original" label while $\beta$ was "forged", and $\|\cdot\|$ denoted Euclidean distance between two vectors.

From weight equation we can find that if the noise level of a segment is close to the average value across the whole picture, the weight $w_{\alpha}$ assigned is small while $w_{\beta}$ is large and vice versa. This meets the requirement of discontinuity preserving. Then it is the turn to discuss smooth constrain. Proper value of interaction penalty $V(\alpha, \beta)$ tolerates local deviations of noise which is affected by illumination or color. There are many forms proposed. For an instance, $V(\alpha, \beta)=\min (K,|\alpha-\beta|)$ or an important function given by 
the Potts model $V(\alpha, \beta)=K \cdot T(\alpha \neq \beta)$, where $T(\cdot)$ is 1 if its argument is true, and otherwise 0 . This penalty function possesses good feature of piecewise smooth, so we used in the experiment. The value of $K$ will be discussed in the later part.

\section{EXPERIMENTAL RESULTS AND DISCUSSIONS}

We created a fake picture by combination of two images downloaded from the popular online photo sharing website Flickr via Photoshop. As it shown in Fig. 7, the leaf was spliced onto the picture to make a forgery and our method successfully detected this area and marked it in white. In order to prove the effectiveness and application of our method, we provide more experimental results in details in this section. At first the appropriate parameter settings will be investigated.

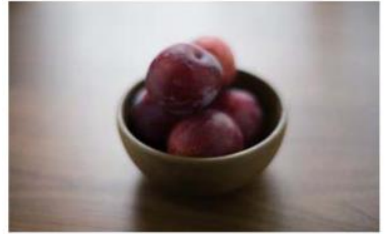

(a)



(c)

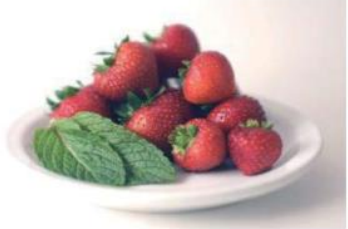

(b)

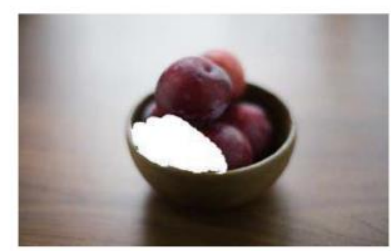

(d)
Fig. 7. Detection result: (a)-(b) source pictures; (c) forged picture; (d) forged area marked in white.

\section{A. Evaluation Criteria}

To evaluate the detection accuracy of our method, we employed a revised Hausdorff distance to measure the distance between the boundaries of detected forged area and that of actual splicing region.

Hausdorff distance [13] was widely used in shape matching measurement and we used a revised version [5] to reflect the "average" other than only the maximum distance of two area. The distance between boundaries of detected area $X\left(x_{1}, x_{2}, \ldots, x_{n}\right)$ and that of actual splicing region $Y\left(y_{1}, y_{2}, \ldots, y_{m}\right) \quad$ is determined by

$D(X, Y)=\max \{d(X, Y), d(Y, X)\}$, where

$$
d(X, Y)=\frac{1}{|X|} \sum_{x \in X} \inf _{y \in Y} d(x, y)
$$

is the cardinality of the set and $d(x, y)$ denotes distance between two pixels.

\section{B. Parameter Settings}

Now we discuss the proper choice of parameter $\$ K \$$ in Potts model which will directly affect experimental results. The Potts model gives the quantitative expression of smooth constrain which keeps the label assignment smoothly cover the whole image. To find the value which produces the best detection result, we composed a fake picture and repeated the experiment in different values of $K$ and the results is shown in Table II and Fig. 8. The highest accuracy appears at $K=0.2$, and smaller or bigger setting leads to lower accuracy and when $K=0.4$ the algorithm failed to exposure the forgery.

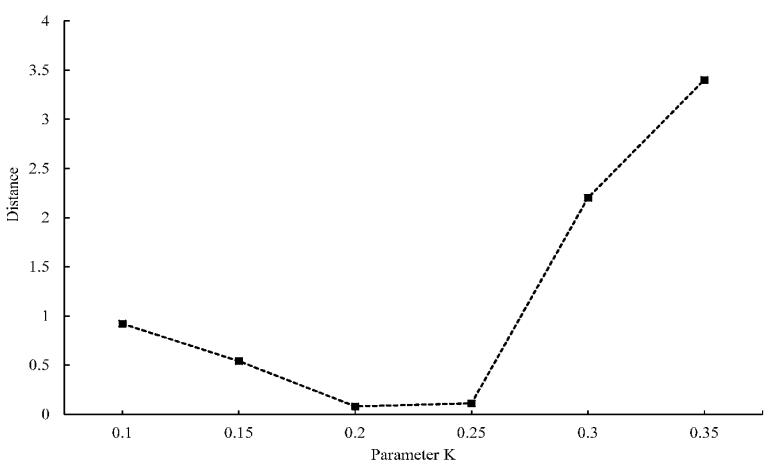

Fig. 8. Different detection accuracy under values of $K$.

TABLE II: DIFFERENT DETECTION ACCURACY UNDER VALUES OF K

\begin{tabular}{cccccccc}
\hline \hline$K$ & 0.1 & 0.1 & 0.2 & 0.2 & 0.3 & 0.3 & 0.4 \\
& 0 & 5 & 0 & 5 & 0 & 5 & 0 \\
\hline$D(X, Y)^{1}$ & 0.9 & 0.5 & 0.0 & 0.1 & 2.2 & 3.4 & $\infty^{2}$ \\
\hline \hline $\begin{array}{l}1 \times 10^{-3} \\
\text { failed to detect the forged area. }\end{array}$ & 2 & 4 & 8 & 1 & & & \\
\end{tabular}

\section{Simulation Results}

In this part we present a simulated forgery case that the noise is added to implanted region. This simulation also reflect a real splicing attack that in order to make the alien area visually resemble the rest part of picture noise may be applied. Since Photoshop is a popular image editing tool, we add noise to picture with provided filters by software. There are two noise distribution options: Gaussian and uniform, and two noise patterns: monochrome and colored. Therefore four combinations are available and the user can alter the noise amount in percentage. The experiment is designed to demonstrate the sensibility of algorithm: what is the lower limit amount of added noise that can be detected by our method. Fig. 9 shows the detection accuracy of four groups, each of which contains five forged pictures. We conclude the effective lower limit for detection is $1.4 \%$ for Gaussian noise and $2.2 \%$ for uniform noise regardless of monochrome or colored noise pattern.

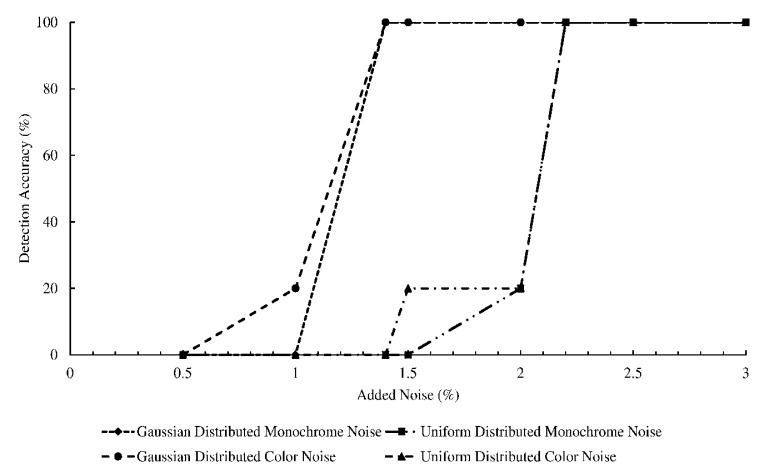

Fig. 9. Finding lower limit amount of added noise that the algorithm can be detected. 


\section{Results}

Two image datasets are prepared to verify the effectiveness of our proposed method. In the first set, all source pictures were taken by a Nikon D7000 DSLR camera and used to make splicing forgeries in combination of different ISO speed seen in Table III. There are 10 forged pictures in the test set. The data in table is the detection accuracy, or true positive rate.

TABLE III: COMBINATION OF ISO SPEED AND RESPECTIVE TP RATE (SOURCE PICTURES ARE TAKEN BY NIKON D7000)

\begin{tabular}{ccccccc}
\hline \multicolumn{7}{c}{ SOURCE PICTURES ARE TAKEN BY NIKON D7000) } \\
\hline ISO & 100 & 200 & 400 & 800 & 1600 & 3200 \\
\hline 100 & $-{ }^{*}$ & 20 & 90 & 100 & 100 & 100 \\
\hline 200 & 20 & - & 40 & 80 & 100 & 100 \\
\hline 400 & 90 & 40 & - & 50 & 100 & 100 \\
\hline 800 & 100 & 80 & 50 & - & 80 & 100 \\
\hline 1600 & 100 & 100 & 100 & 80 & - & 70 \\
\hline 3200 & 100 & 100 & 100 & 100 & 70 & - \\
\hline \hline
\end{tabular}

* Not verified in experiment.

The ISO speed setting in camera is discrete without same interval and we find the higher TP rate appears at combination of two ISO speed with big gap. In order to see this phenomenon clearly, we can see Fig. 10. The horizontal axis is marked by interval stop(s) which denotes the interval ISO speed. For instance, the interval stop of ISO 100 and 200 is 1 , this is the same with ISO 1600 and 3200, while that of ISO 200 and 1600 is 3 . The average TR rate is calculated from Table III and Table IV. We conclude that our method show good performance in two or more interval stops.

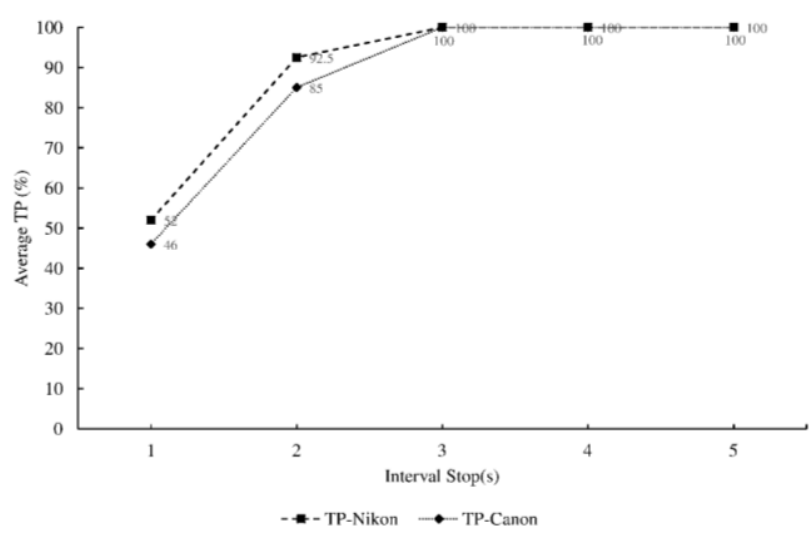

Fig. 10. TP rate in different interval stop(s).

TABLE IV: COMBINATION OF ISO SPEED AND RESPECTIVE TP RATE (SOURCE PICTURES ARE TAKEN BY CANON 550D)

\begin{tabular}{ccccccc}
\hline \hline ISO & 100 & 200 & 400 & 800 & 1600 & 3200 \\
\hline 100 & ${ }^{*}$ & 30 & 80 & 100 & 100 & 100 \\
\hline 200 & 30 & - & 20 & 80 & 100 & 100 \\
\hline 400 & 80 & 20 & - & 40 & 90 & 100 \\
\hline 800 & 100 & 80 & 40 & - & 60 & 90 \\
\hline 1600 & 100 & 100 & 90 & 60 & - & 80 \\
\hline 3200 & 100 & 100 & 100 & 90 & 80 & - \\
\hline \hline
\end{tabular}

* Not verified in experiment.

The second experiment is to verify the effectiveness of detecting forgery in pictures combined from two different cameras. And in the paper we just show an extremely hard situation when the source pictures are taken in same ISO speed. Two cameras are Nikon D7000 and Canon 550D respectively. And 10 forged images in the set are used to test.
The TP rate is shown in Fig. 11. And the accuracy increases as the ISO speed raises. The reason is that the image processing ability of two camera models is not the same. In lower ISO speed, less noise appears in the picture and this processing difference is small, therefore the TP rate is very low at $10 \%$. While in high ISO settings, the method shows effectiveness again. Note that in real situation, the ISO of two source pictures may not the same, only one interval stop will high enhance the accuracy as it is shown in the first experiment.

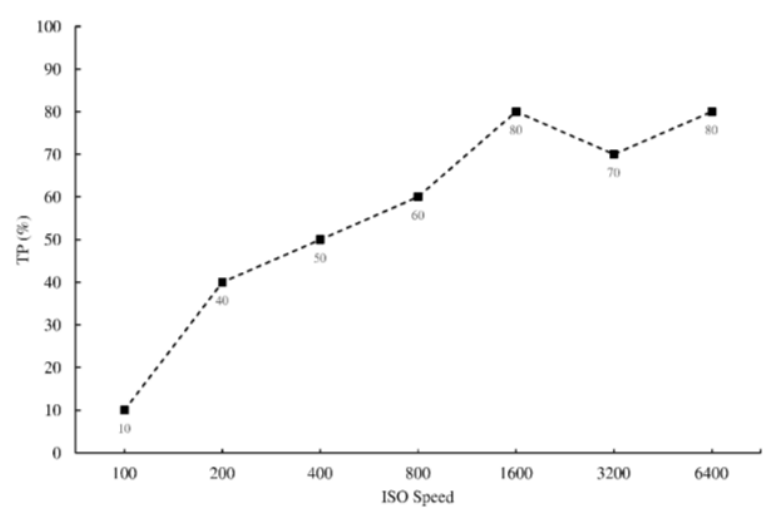

Fig. 11. TP rate in different ISO speed.

\section{CONCLUSIONS}

We have presented in this paper a novel method to exposure splicing forgeries by detecting noise discrepancies. The noise inevitably exists in the picture captured by camera and under different circumstances such as ISO speed and exposure time the noise level and patterns varies. This fact can be therefore used to identify the region shows inconsistencies with the rest of image in terms of noise amount or patterns. With the help of SLIC superpixels image segmentation algorithms the meaningful segments are recognized and noise estimation of each segment measures the noise pattern and level. And eventually the method of energy-based graph cuts labels the splicing regions. Experimentally we discussed the proper parameter choice, demonstrated the good detection result in different scenarios, and proved the robustness and showed advantage of this algorithm by comparing to existing methods. The future work will include developing new ways to measure and evaluate the noise patterns to improve our methods.

\section{ACKNOWLEDGMENT}

The authors would like to thank the referees for their valuable comments. This research was supported in part by Research Committee of the University of Macau (MYRG134-FST11-PCM, MYRG181-FST11-PCM) and the Science and Technology Development Fund of Macau SAR (Project No. 008/2013/A1).

\section{REFERENCES}

[1] S. Chen, B. Mulgrew, and P. M. Grant, "A clustering technique for digital communications channel equalization using radial basis function networks," IEEE Trans. on Neural Networks, vol. 4, pp. 570-578, July 1993

[2] B. Mahdian and S. Saic, "Blind authentication using periodic properties of interpolation," IEEE Transactions on Information Forensics and Security, vol. 3, no. 3, pp. 529-538, 2008. 
[3] A. C. Popescu and H. Farid, "Exposing digital forgeries by detecting traces of resampling," Signal Processing, IEEE Transactions on, vol. 53, no. 2, pp. 758-767, 2005 .

[4] P. Kakar, N. Sudha, and W. Ser, "Exposing digital image forgeries by detecting discrepancies in motion blur," IEEE Transactions on Multimedia, vol. 13, no. 3, pp. 443-452, 2011.

[5] S. Hamdy, H. El-Messiry, M. Roushdy, and E. Kahlifa, "Quantization table estimation in jpeg images," International Journal of Advanced Computer Science and Applications, vol. 1, no. 6, pp. 17-23, 2010.

[6] H. M. Gou, A. Swaminathan, and M. Wu, "Noise features for image tampering detection and steganalysis," in Proc. IEEE International Conference on Image Processing, 2007, vol. 6, pp. VI97-VI100.

[7] L. G. Shapiro and G. C. Stockman, Computer Vision, New Jersey: Prentice-Hall, 2011.

[8] R. Achanta, A. Shaji, K. Smith, A. Lucchi, P. Fua, and S. Susstrunk, "Slic superpixels compared to state-of-the-art superpixel methods," IEEE Transactions on Pattern Analysis and Machine Intelligence, vol. 34, no. 11, pp. 2274-2282, 2012.

[9] J. Y. Fan, H. Cao, and A. C. Kot, "Estimating exif parameters based on noise features for image manipulation detection," IEEE Transactions on Information Forensics and Security, vol. 8, no. 4, pp. 608-618, 2013.

[10] H. M. Gou, A. Swaminathan, and M. Wu, "Intrinsic sensor noise features for forensic analysis on scanners and scanned images," IEEE Transactions on Information Forensics and Security, vol. 4, no. 3, pp. 476-491, 2009.

[11] Y. Boykov, O. Veksler, and R. Zabih, "Fast approximate energy minimization via graph cuts," Pattern Analysis and Machine
Intelligence, IEEE Transactions on, vol. 23, no. 11, pp. 1222-1239, 2001.

[12] V. Hodge and J. Austin, "A survey of outlier detection methodologies," Artificial Intelligence Review, vol. 22, no. 2, pp. 85-126, 2004.

[13] N. Otsu, "Threshold selection method from gray-level histograms," Automatica, vol. 11, pp. 285-296, 1975.

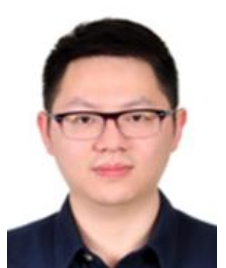

Bo Liu received his B.M. degree in information management and systems from University of International Relations, China in June 2010 and his M.Sc. degree in e-commerce technology from University of Macau, Macau in Jan 2014. His current research interests include forgery detection in digital image, image processing, pattern recognition and computer vision.

Chi-Man Pun received the B.Sc. and M.Sc. degrees from the University of Macau, Taipa, Macau, in 1995 and 1998, respectively, and the Ph.D. degree in computer science and engineering from the Chinese University of Hong Kong, Shatin, Hong Kong, in 2002. He currently is an associate professor with the Department of Computer and Information Science, University of Macau. His research interests include digital watermarking, multimedia compression, analysis and processing, pattern recognition, and computer vision. 